\title{
Registro geométrico y modelado tridimensional de la cueva de Praileaitz I (Deba, Gipuzkoa)
}

\author{
Debako Praileaitz I kobazuloaren erregistro geometrikoa eta hiru \\ dimentsioko modelaketa (Deba, Gipuzkoa)
}

\section{Recording the geometrical form and modelling of Praileaitz I cave in Deba (Gipuzkoa)}

PALABRAS CLAVE: Cuevas, Documentación geométrica, Topografía, Fotogrametría, Modelado 3D, Anáglifos. GAKO-HITZAK: Kobazuloak, Dokumentazio geometrikoa, Topografia, Fotogrametria, Hiru dimentsioko modelaketa, Anaglifo irudiak. KEYWORDS: Caves, Geometric documentation, Surveying, Photogrammetry, 3D modeling, Anaglyphs.

\section{Álvaro RODRíGUEZ-MIRANDA, Ane LOPETEGI-GALARRAGA, José M. VALLE-MELÓN(1)}

\section{RESUMEN}

El presente artículo describe dos modelos tridimensionales de la cueva de Praileaitz I, el primero de ellos se realizó en el año 2003 utilizando técnicas topográficas, mientras que el segundo se ha realizado en 2015 recuperando la documentación fotográfica que se obtuvo en ese mismo trabajo de 2003. La presentación de estos dos modelos permite reflexionar sobre la evolución de las técnicas de documentación geométrica de cavidades en los últimos años y analizar su potencial en el momento actual.

\section{LABURPENA}

Artikulu honetan Praileaitz I kobazuloaren hiru dimentsioko bi eredu desberdin deskribatzen dira. Haietako lehena 2003 urtean egin zen teknika topografikoak erabiliz eta bigarrena 2015 urtean, 2003ko lanean bildu zen argazki sorta berrerabiliz. Bi ereduen ezaugarriak aztertuz, azken urteetako kobazuloen dokumentazio geometrikoaren bilakaera hausnartu daiteke eta gaurko uneetan eskaintzen duen potentzialaren analisia egin.

\section{ABSTRACT}

This paper describes two three-dimensional models of Praileaitz I cave, the first one was generated in 2003 by means of surveying techniques whereas the second was created in 2015 with the photographs taken in the previous work. These two models allow us to reflect on the evolution of the techniques applied to the geometrical documentation of caves during the last years and to analyze what the potential of these techniques is nowadays.

\section{1.- INTRODUCCIÓN}

A finales del año 2003 y ante el riesgo de destrucción de la cueva de Praileaitz I (Deba, Gipuzkoa) debido a las labores de explotación de la vecina cantera, el Servicio de Patrimonio de la Excma. Diputación Foral de Gipuzkoa consideró necesario documentar geométricamente el espacio y generar un modelo tridimensional (Laboratorio de Documentación Geométrica del Patrimonio, 2004). Transcurrida ya más de una década desde aquel momento, la elaboración del presente monográfico que recoge las diferentes actuaciones realizadas en la cueva desde el año 2000 nos ofrece la posibilidad de revisar dicho trabajo y ponerlo en relación con una metodología general de documentación de cavidades que puede ser replicable en muchos otros casos, siempre que se tengan en cuenta las correspondientes adaptaciones a los objetivos de cada trabajo, las particularidades de cada cueva y las características derivadas de la aplicación de los avances tecnológicos y las nuevas herramientas de medida y modelado geométrico.

Por aquel entonces, la morfología visible del interior de la cueva correspondía a la sala principal -vestíbulode unos 11 metros de altura y con una base de unos 4 metros de ancho por 8 metros de largo; esta primera cavidad daba acceso a otras dos salas laterales de menor tamaño situadas respectivamente a la derecha y a la izquierda de la entrada. Una línea de puntos marcada en las paredes reflejaba la altura inicial del suelo que ya había sido rebajado entre uno y dos metros según la zona (Fig. 1). La estancia a mano izquierda continuaba mediante una abertura que conectaba con una nueva sala (Fig. 2); no obstante, debido a que toda esta zona se encontraba aún muy colmatada, se decidió no incluirla en el modelo tridimensional a generar. Como es sabido, en los años

\footnotetext{
(1) Laboratorio de Documentación Geométrica del Patrimonio - Grupo de Investigación en Patrimonio Construido, Universidad del País Vasco/Euskal Herriko Unibertsitatea UPV/EHU. Aulario de las Nieves, edificio de Institutos Universitarios, B4 c/ Nieves Cano 33. 01006 , Vitoria-Gasteiz, España. Idgp@ehu.eus
} 


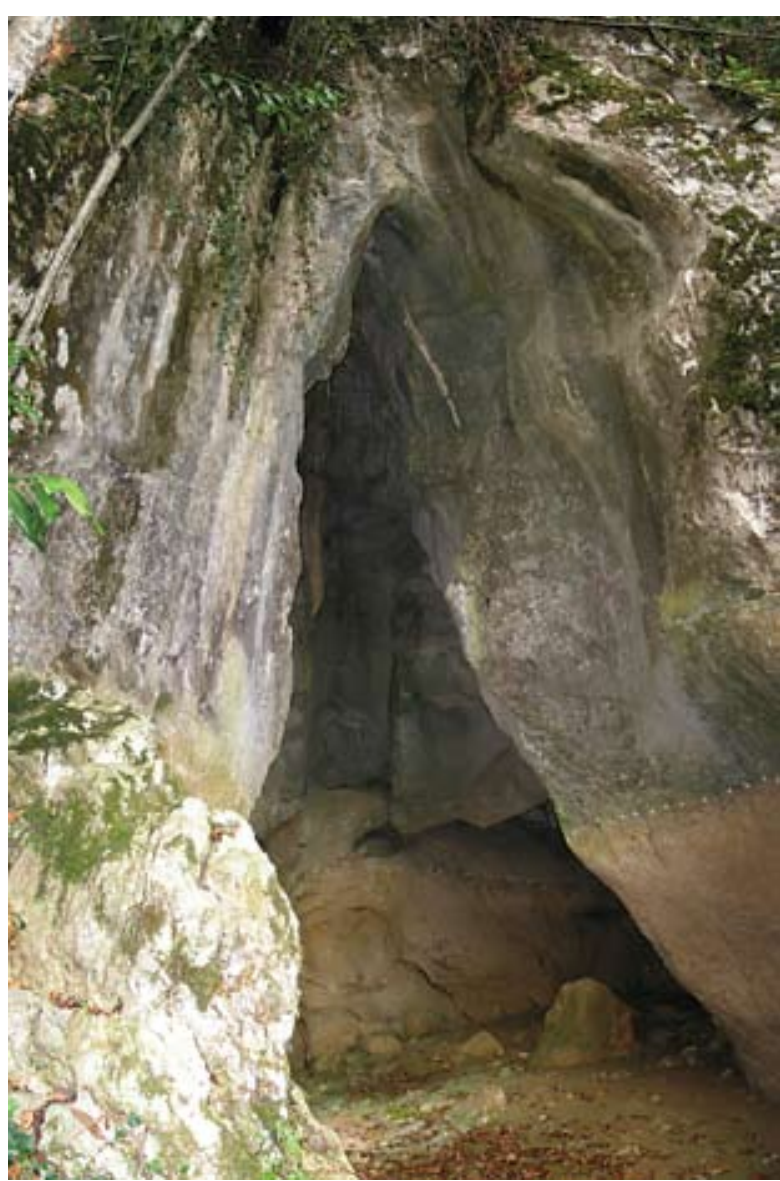

Fig. 1. Entrada a la cueva a finales de 2003, el cambio de tonalidad de la roca y la línea de puntos blancos indican el nivel del suelo original. / Entry of the cave by the end of 2003, the change in the tonality of the stone and the line of white dots show the original level of the ground.

posteriores se descubrieron representaciones parietales en zonas más profundas de la cavidad pero, en 2003, aún se desconocía su existencia.

Los objetivos que se marcaron para el trabajo de documentación y modelado se resumen en la siguiente lista:

a) Documentación fotográfica del estado inicial. Con el fin de apreciar los detalles tridimensionales de la cavidad, las fotografías se tomarían formando pares estereoscópicos y se prepararía una colección de imágenes en anáglifo (rojo-cian) para su difusión.

b) Documentación geométrica mediante topografía clásica del volumen de la cueva junto con su entorno inmediato. El sistema de coordenadas utilizado sería el correspondiente a la cartografía oficial ${ }^{1}$, de

\footnotetext{
${ }^{1}$ En 2003, la cartografía oficial se representaba en proyección UTM (huso 30) en el sistema de referencia denominado ED-50. A partir de 2007 se produjo un cambio: si bien la proyección seguía siendo UTM, el sistema de referencia utilizado pasó a ser el ETRS89; para la zona de estudio, este cambio supone un desplazamiento en las coordenadas de aproximadamente 100 metros en la dirección X y 200 en la dirección Y.
}

esta forma, los productos generados se podrían poner en relación con otros elementos como la mencionada cantera.

c) Modelado alámbrico (es decir, definido mediante las líneas que marcan las aristas más representativas) que, posteriormente, serviría de base para la generación de las superficies que conforman la cueva. A partir de este modelo 3D en el entorno CAD se prepararía una colección de planos: plantas, alzados, secciones y perspectivas. Asimismo, el modelo tridimensional también se exportaría para su exploración interactiva.

d) Por otro lado, tanto la información recogida como los resultados se documentarían convenientemente y se prepararían para que pudieran seguir siendo utilizados en el futuro.

\section{2.- DOCUMENTACIÓN GEOMÉTRICA DE CAVI- DADES}

Por regla general, los trabajos topográficos en cuevas tienen algunas particularidades que es preciso considerar:

- Desde el punto de vista geométrico, se trata de una documentación plenamente tridimensional en el que el elemento a documentar envuelve completamente al visitante, la morfología de las formaciones geológicas puede llegar a revestir una complejidad elevada requiriendo, por lo tanto, recurrir a sistemas de registro que proporcionen un gran nivel de detalle. Por otro lado, los puntos desde donde se pueden realizar las observaciones son limitados, lo que unido a la abundancia de recovecos puede ocasionar que existan zonas ocultas. Asimismo, también es habitual tener que salvar pa-

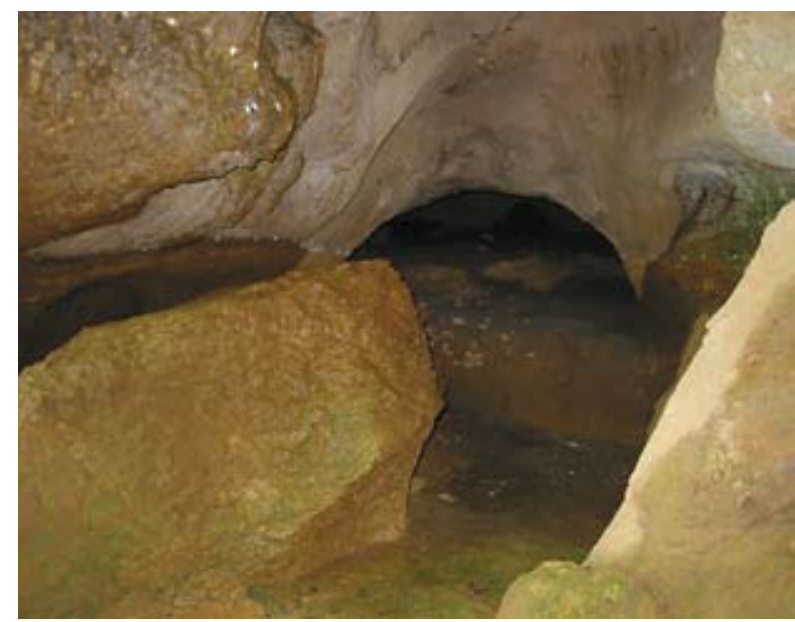

Fig. 2. Estado semicolmatado de la boca de acceso a la cámara que continúa a la izquierda (según se accede) de la sala principal. / Semi-clogged access to the chamber that gives continuity to the cave on the left hand side of the entrance. 
sos estrechos o bruscos desniveles que, además de suponer dificultades de tránsito y condiciones especiales de seguridad en el trabajo, pueden afectar negativamente a la precisión de las medidas.

- En general, será necesario disponer de sistemas de iluminación adicional. Si los puntos a medir los selecciona el técnico directamente en campo es preciso que los vea y si se recurre a la fotogrametría la iluminación ha de ser suficientemente homogénea para que los diferentes elementos sean identificables en varias fotografías. Por su parte, los escáneres láser son sistemas activos por lo que pueden trabajar a oscuras; sin embargo, al igual que sucede con las estaciones totales, las distancias medidas pueden verse afectadas por la humedad, tanto del ambiente como la que impregna las superficies. Por supuesto, la señal GPS no llega al interior de las cuevas por lo que este tipo de equipos sólo pueden utilizarse en el exterior.

- Respecto al recorrido, los itinerarios topográficos se suelen planificar para que comiencen y terminen en puntos de coordenadas conocidas, de esta manera se puede comprobar fácilmente que: (1) los errores acumulados a lo largo de todos los puntos intermedios no superan el límite establecido para la cartografía a realizar, y (2) que no existan medidas erróneas. Sin embargo, cuando se trata de galerías que terminan en algún lugar en el interior de la montaña no existe un conocimiento previo de las coordenadas del punto final.

- A todo lo anterior hay que sumar el principio de que el trabajo de documentación sea lo menos agresivo posible para la propia cueva, los espeleotemas, las manifestaciones artísticas o los restos arqueológicos lo que, por ejemplo, exige que se reduzca la cantidad de señales de referencia que se instalan de manera permanente, en especial, si dichas señales suponen una alteración de dichos elementos.

Al hablar de los diferentes tipos de cartografía de cavidades es imperativo comenzar por los trabajos que tradicionalmente han venido realizando los grupos espeleológicos (véase, por ejemplo, ALCALÁ et al., 2013). Se trata de una documentación con personalidad propia y de gran expresividad que saca el máximo partido a unos medios de trabajo sencillos (principalmente: distanciómetro de mano, brújula y clinómetro) y adaptada a las condiciones de tránsito de la cueva que, en ocasiones, revisten de una gran dificultad. No es infrecuente que este tipo de representaciones se sigan utilizando para la documentación general de las cavidades y que, complementadas con la documentación gráfica desarrollada por los propios equipos de arqueología y los calcos (para el arte parietal), sirvan para ilustrar solventemente las necesidades de un trabajo arqueológico en cuevas, tal como puede comprobarse en numerosos trabajos (BOTELLA et al., 2006; RUIZ, 2010, etc.).
El recurso al equipamiento topográfico suele venir motivado por un incremento en las necesidades de precisión o densidad de detalles, así como por el interés en capturar la morfología de la cavidad tridimensionalmente. En un principio, dicho equipamiento topográfico consistía en estaciones totales, como puede verse en algunos ejemplos de comienzos de la década del 2000, caso de Ojo Guareña (GÓMEZ-BARRENA et al., 2001) o la Cueva de los Murciélagos (CÓRDOBA et al., 2004); siendo éste también el instrumental que se empleó en la cueva de Praileaitz I (LOPETEGl et al., 2004). El motivo de que esta técnica se popularizase a partir de esta fecha fue debido a la aparición de las estaciones de medida sin prima, las cuales podían determinar las coordenadas de puntos visados directamente sin necesidad de tener que situar en ellos un prisma reflector, lo que permitió no sólo aumentar la velocidad de captura de datos sino también la determinación de puntos en techos y lugares inaccesibles. Anteriormente a la llegada de estos equipos de medida, la determinación de los puntos inaccesibles se debía realizar mediante intersección angular y requería observar el mismo punto desde varias posiciones, lo cual acarreaba problemas en la identificación de los elementos y suponía un proceso de captura más engorroso y lento.

Por su parte, la aplicación de la fotogrametría estereoscópica a la medida de cuevas se dio a conocer -en nuestro entorno- con el trabajo realizado en Altamira a finales de la década de los setenta (GARCÍA, LLANOS, 1981) por el personal del Instituto Geográfico Nacional, equipo que también realizó otros trabajos posteriores como la cueva de Lluera en Asturias (FORTEA, 1990). En estos casos, los pares estereoscópicos se utilizaban para su restitución, es decir, para el dibujo manual de líneas y curvas de nivel. Siguiendo este sistema, podemos encontrar otros ejemplos como la mencionada Ojo Guareña (GÓMEZ-BARRENA et al., 2001) o la Cova Rivasals en Castellón (BUCHÓN et al., 2002), siendo esta última un ejemplo de aplicación temprana de los Sistemas de Información Geográfica al manejo de la información extraída y de la obtención de ortofotografías.

Con el desarrollo de la fotografía digital (más concretamente, gracias a las técnicas de medida de puntos por correlación, es decir, por identificación automática en varias imágenes) y la aparición en el mercado de los escáneres tridimensionales se dio comienzo a una nueva etapa en la cual era posible documentar de forma masiva y rápida grandes superficies con un elevado nivel de detalle. La discusión sobre cuál de estas dos metodologías es más ventajosa está planteada desde hace una década (FRYER et al., 2005) y, afortunadamente, todavía sigue activa gracias a los avances tecnológicos que van surgiendo y que han ido mejorando sustancialmente las posibilidades de documentación en los últimos años. De hecho, en la actualidad es una práctica habitual utilizar una combinación de ambas, de forma que el escáner láser proporciona la geometría y la fotografía digital de alta resolución aporta la textura (GRUSSENMEYER et al., 


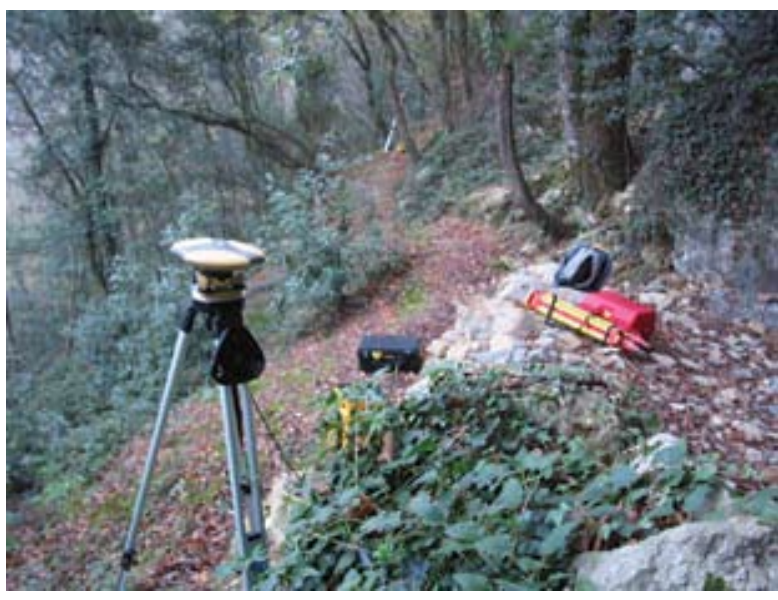

Fig. 3. Receptor GPS observando en la entrada de la cueva. Nótese que el horizonte de observación está parcialmente cubierto entre la vegetación y la propia ladera, por este motivo, y con el fin de alcanzar una precisión centimétrica en estos puntos, se prolongó el periodo de captura de datos hasta las 3 horas por punto. / GPS receiver collecting data in front of the cave. Do note that the sky view is partly hidden by trees and the own hillside, for that reason and with the aim of obtaining centimetric accuracy, each point was observed for 3 hours.

2010; LERMA et al., 2010; GONZÁLEZ-AGUILERA et al, 2011; CORCHÓN et al., 2011).

También hay que tener en cuenta el hecho de que las cuevas pueden requerir un nivel variable de detalle en función de la complejidad o el interés de las estructuras geológicas de cada zona o la presencia de arte parietal que, en ocasiones (como sucede con las incisiones), pueden demandar resoluciones sub-milimétricas. En estos casos, la combinación de instrumental es preceptiva, por ejemplo: varios tipos de escáneres láser adaptados a diferentes rangos de distancias y precisiones (WARZÉE et al., 2008) o la utilización de un escáner láser para la geometría general de la cavidad y técnicas fotogramétricas de alta resolución para los elementos de detalle (CABRELLES, LERMA, 2013).

En cualquier caso, una vez generados los modelos tridimensionales, las posibilidades de explotación son muy variadas, abarcando desde la obtención de productos cartográficos hasta su uso para la difusión al público, tal como muestra el ejemplo de Santimamiñe (BARRERA, BAEZA, 2010). Por supuesto, las técnicas de documentación no sólo son aplicables a la estructura geológica sino que también lo son al seguimiento de las excavaciones arqueológicas (RIOS et al, 2012; NÚÑEZ et al., 2013).

\section{3.- MODELADO 3D DE LA CUEVA DE PRAI- LEAITZ I}

Visto el contexto desarrollado en la sección anterior, pasamos a describir el trabajo realizado en la cueva de Praileaitz I en 2003 y dejamos para un apartado posterior

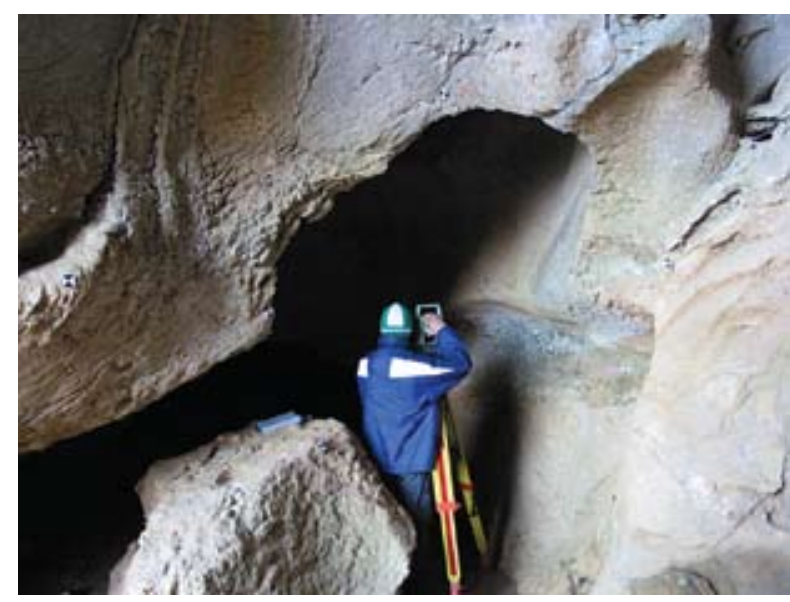

Fig. 4. Medida de la geometría de la cueva mediante estación total / Surveying of the cave by total station.

el análisis de la información capturada a la luz de las posibilidades tecnológicas actuales.

En primer lugar, se estableció la red de referencia topográfica a partir de la cual se realizarían las medidas de los diferentes elementos que conforman la cueva. Para ello, se comenzó situando tres bases (materializadas mediante un clavo sobre una estaca de madera hincada en el terreno) en el exterior de la entrada que se observaron utilizando técnicas GPS (Fig. 3) de forma que se obtuvieron sus coordenadas en el sistema cartográfico oficial. En segundo lugar, situándonos en uno de estos puntos y mediante medidas con estación total topográfica se dotó de coordenadas a otra base situada en el interior de la cueva desde la cual se abarcaba tanto el vestíbulo como las salas contiguas, posteriormente se estacionó en esta base del interior desde la cual se midieron las coordenadas de un conjunto de 36 dianas que se habían colocado por todo el recorrido en planta de la cueva (aproximadamente a un metro y medio de altura). Estas dianas tenían un tamaño de $4 \times 4 \mathrm{~cm}$, estaban hechas de papel plastificado, se fijaron a las paredes mediante un adhesivo plástico y fueron retiradas al finalizar el trabajo.

La metodología seleccionada para el registro de la geometría de la cueva fue la medida directa desde estación total (Fig. 4), es decir, que el operario iba seleccionando los puntos que definen la geometría directamente en campo, los visaba a través del anteojo del equipo y registraba sus coordenadas. Para cubrir la totalidad de la zona se realizaron seis estacionamientos, obteniendo las coordenadas y orientación de la estación total mediante intersección inversa a las dianas de la red de referencia, tras lo cual, las coordenadas medidas de los puntos ya se encontraban en el sistema del trabajo.

Los datos capturados en campo mediante la estación total correspondían a las líneas que definían los perímetros más destacados y un conjunto de puntos en el interior de cada recinto que, posteriormente, se utilizarían para generar las superficies. El nivel de detalle de 

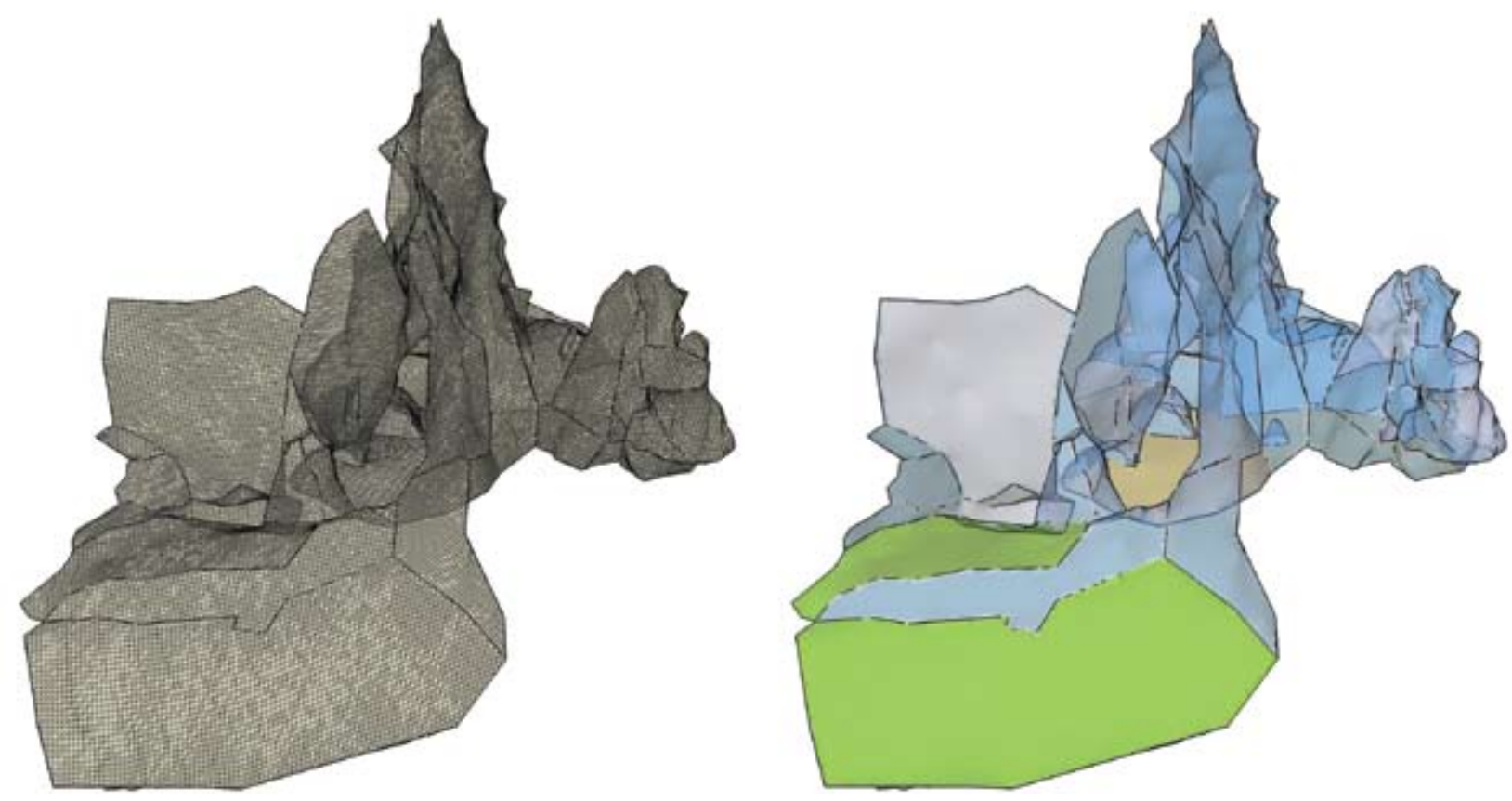

Fig. 5. Modelo mallado. La imagen de la izquierda muestra una vista de las mallas generadas sin diferenciarlas por el tipo de superficie que representan, la imagen de la derecha por el contrario identifica las zonas de roca, suelo interior y suelo exterior. / Meshed model. The image on the left shows a view of the meshes generated without material differentiation; on the contrary, the image on the right identifies the surfaces of stone and the ground inside and outside the cave.

los perímetros y de densidad de los puntos de relleno se estableció pensando en que la representación final correspondería a un modelo mallado en el que las mallas tuviesen un tamaño de $10 \times 10 \mathrm{~cm}$ (Fig. 5).

A partir de este modelo se confeccionaron varias salidas gráficas (planos) con el fin de representar diferentes aspectos de la cavidad.

\section{4.- REUTILIZACIÓN DE LA INFORMACIÓN PARA LA GENERACIÓN DE NUEVOS PRODUCTOS}

La representación de la cueva se completó con una colección de imágenes estereoscópicas preparadas en forma de anáglifos que permiten analizar con gran detalle el relieve de las estructuras geológicas (Fig. 6). Para la captura se diseñó un sistema que mediante una barra ajustable a un trípode permitía colocar dos cámaras fotográficas, de forma que la toma del par estereoscópico pudiese hacerse de forma más ágil y que la calidad visual de los anáglifos generados fuese mejor ya que se garantizaba el paralelismo de las tomas (Fig. 7).

En el año 2015, se decidió recuperar esta colección de fotografías -182 imágenes- y probar a reprocesarla utilizando las herramientas informáticas de modelado disponibles en la actualidad, de esta manera podríamos comprobar su potencial para la generación de nuevos productos.

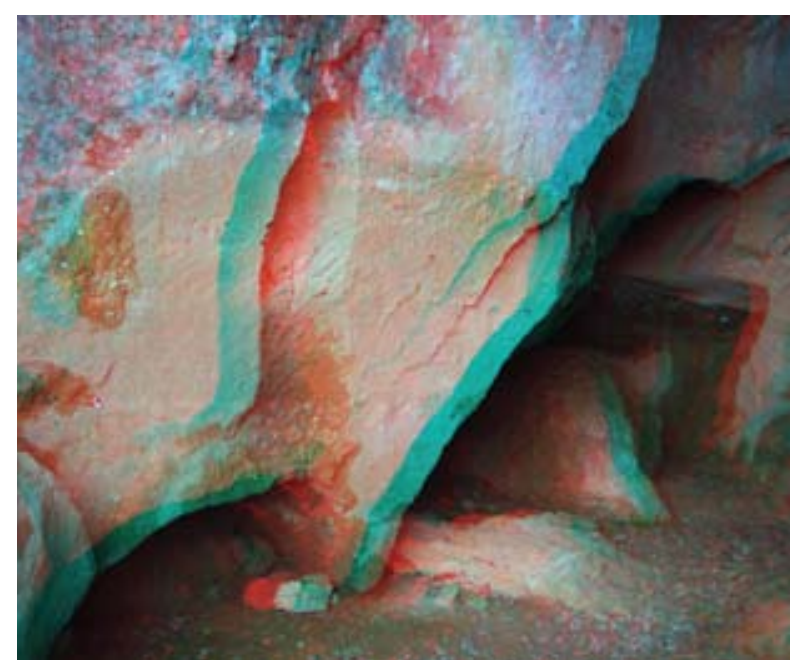

Fig. 6. Uno de los anáglifos generados del interior de la cueva. / One of the anaglyphs taken inside the cave.

En concreto, se utilizó el programa Photoscan $®$ que permite orientar automáticamente las imágenes identificando puntos comunes entre las diferentes imágenes (Fig. 8). Una vez orientadas las imágenes se procedió a calcular una nube de puntos densa (Fig. 9) en la que ya es posible identificar, a grandes rasgos, la morfología de la cueva.

De manera similar al procedimiento que se desarrolló en 2003, de la nube de puntos se pasa al modelo 


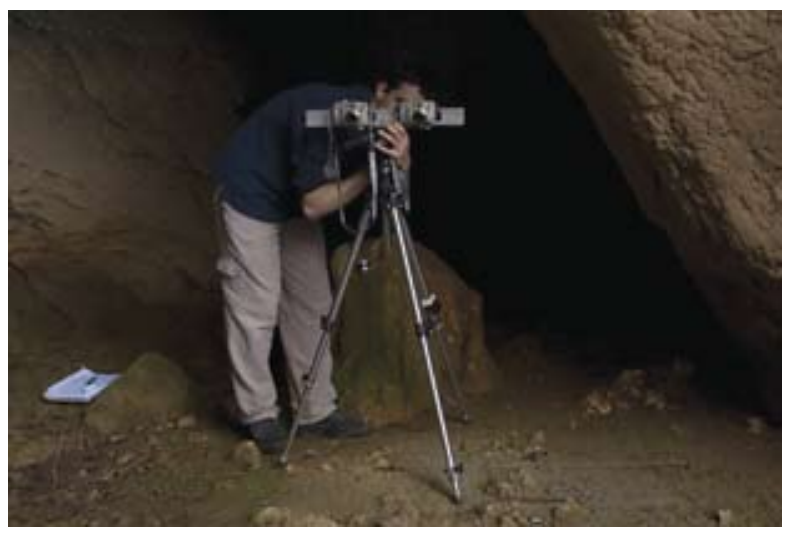

Fig. 7. Bicámara empleada para la toma de las fotografías que se utilizaron para la generación de los anáglifos. / Pair of cameras employed to capture the photographs used for the generation of the anaglyphs. de superficies. En este caso, el mallado obtenido es del tipo TIN (Triangulated Irregular Network) -véase la comparación de ambos tipos en la Figura 10-. Al tratarse de un modelado realizado a partir de fotografías es posible aplicar las texturas al modelo de superficies, obteniendo así una representación de gran realismo. Aunque el aspecto general es bastante evocador, cabe mencionar que el modelo generado presenta algunas limitaciones debidas a las características de las tomas (todas desde el suelo) y los recovecos existentes que generan defectos en el modelado y en el recubrimiento de texturas en ciertas zonas.

En todo caso, este nuevo modelo 3D es adecuado para su exploración interactiva y, asimismo, sirve para generar nuevos productos cartográficos como ortoimágenes, secciones, etc. (Fig. 11).

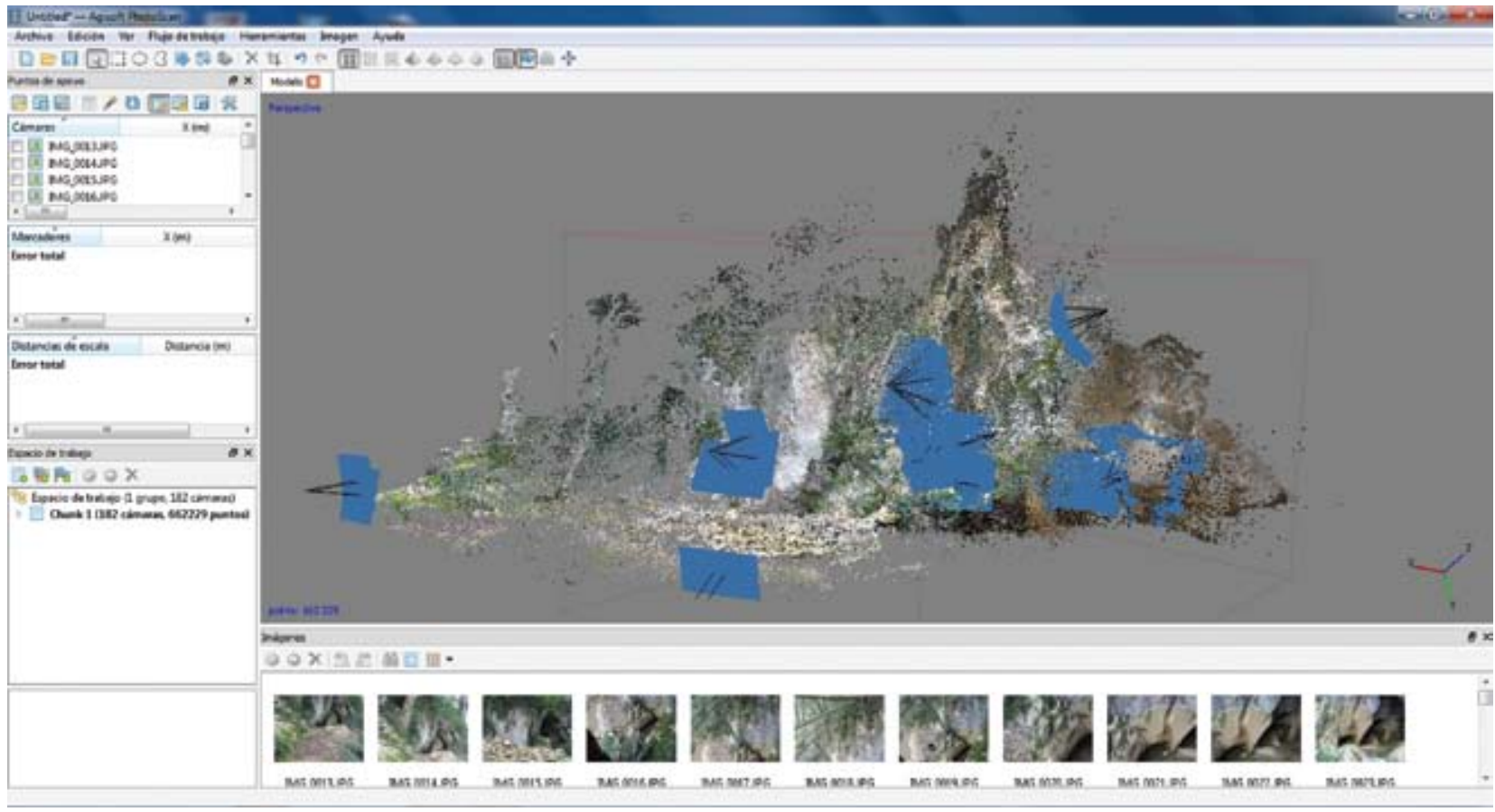

Fig. 8. Orientación automática de las fotografías utilizadas en los anáglifos (cuadros azules) en una vista tridimensional en la que también se representan los puntos comunes localizados en las imágenes. / Automatic orientation of the photographs originally used in the anaglyphs (blue squares) in a 3D view in which are also represented the common points located in the pictures.

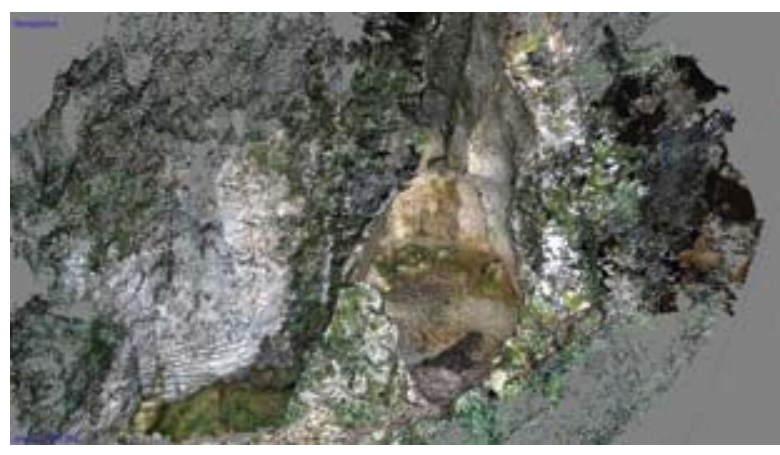

Fig. 9. Nube de puntos densa generada por correlación entre las imágenes que, originalmente, se tomaron para la generación de los anáglifos. / Dense point cloud generated by means of image matching with the pictures originally taken for the anaglyphs. 


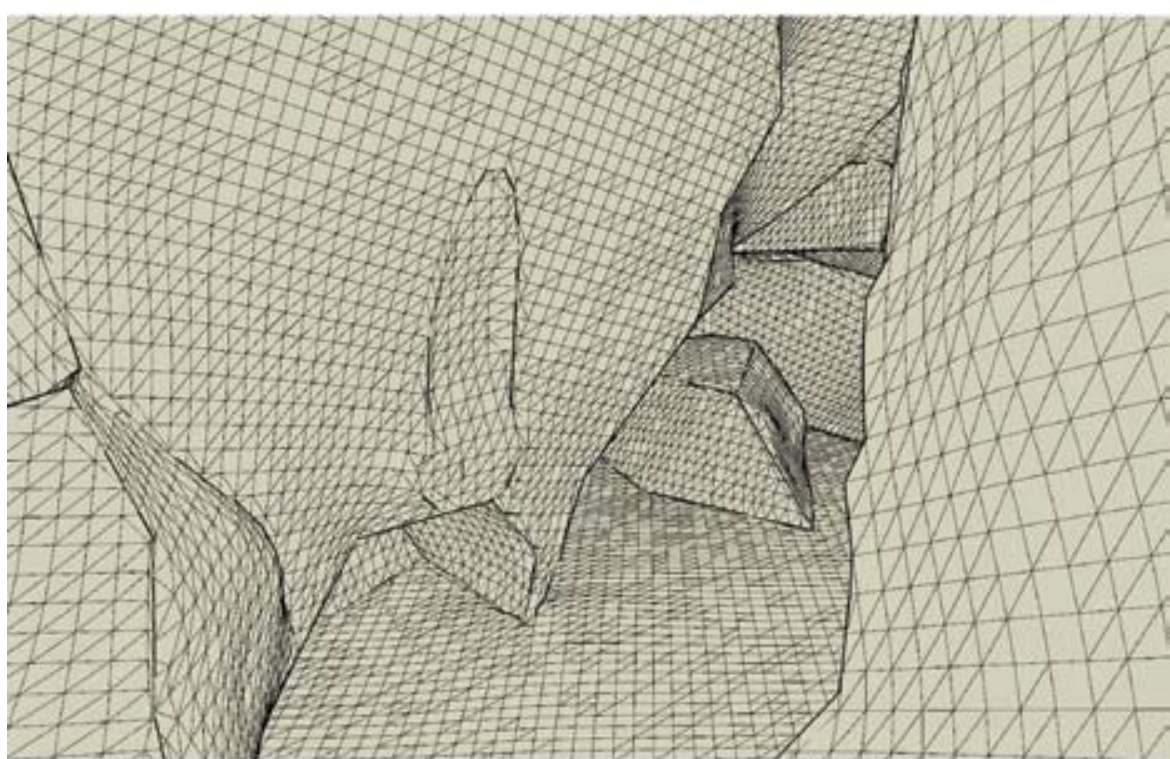

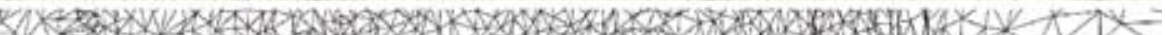
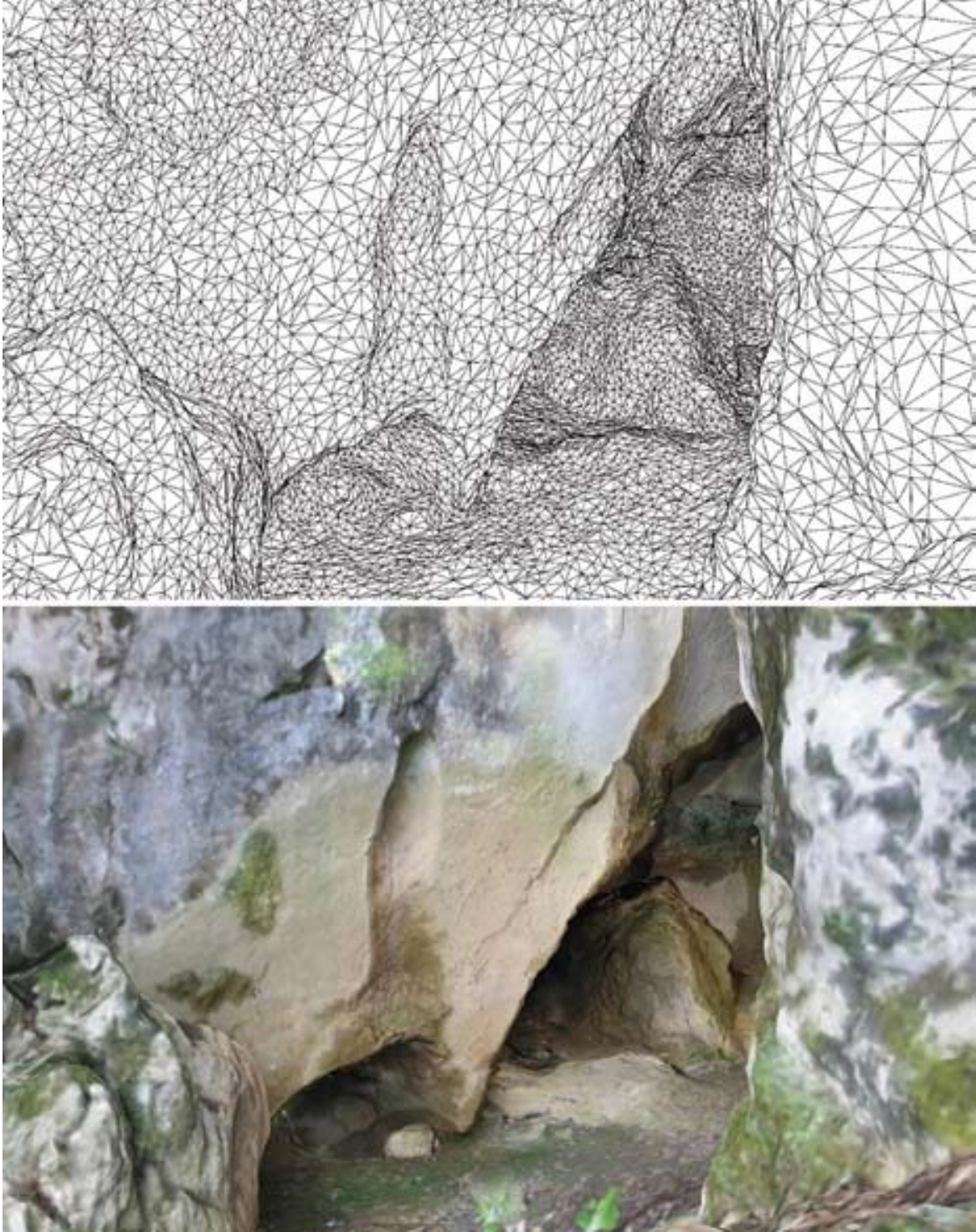

Fig. 10. Comparativa del mallado regular realizado en 2003 (arriba) y el mallado irregular generado en el procesado de modelado automático de las fotografías tomadas para los anáglifos (centro). La imagen inferior muestra la misma zona con texturas fotográficas. / Comparison of the regular mesh made in 2003 (up) and the irregular mesh generated by the automatic processing of the photographs taken for the anaglyphs (centre). The image at the bottom shows the same area with photographic texture. 
Fig. 11. Vista perspectiva de una sección (línea roja) del nuevo modelo tridimensional con texturas fotográficas. / Perspective view of a longitudinal section (red line) of the new three-dimensional model with photographic textures.

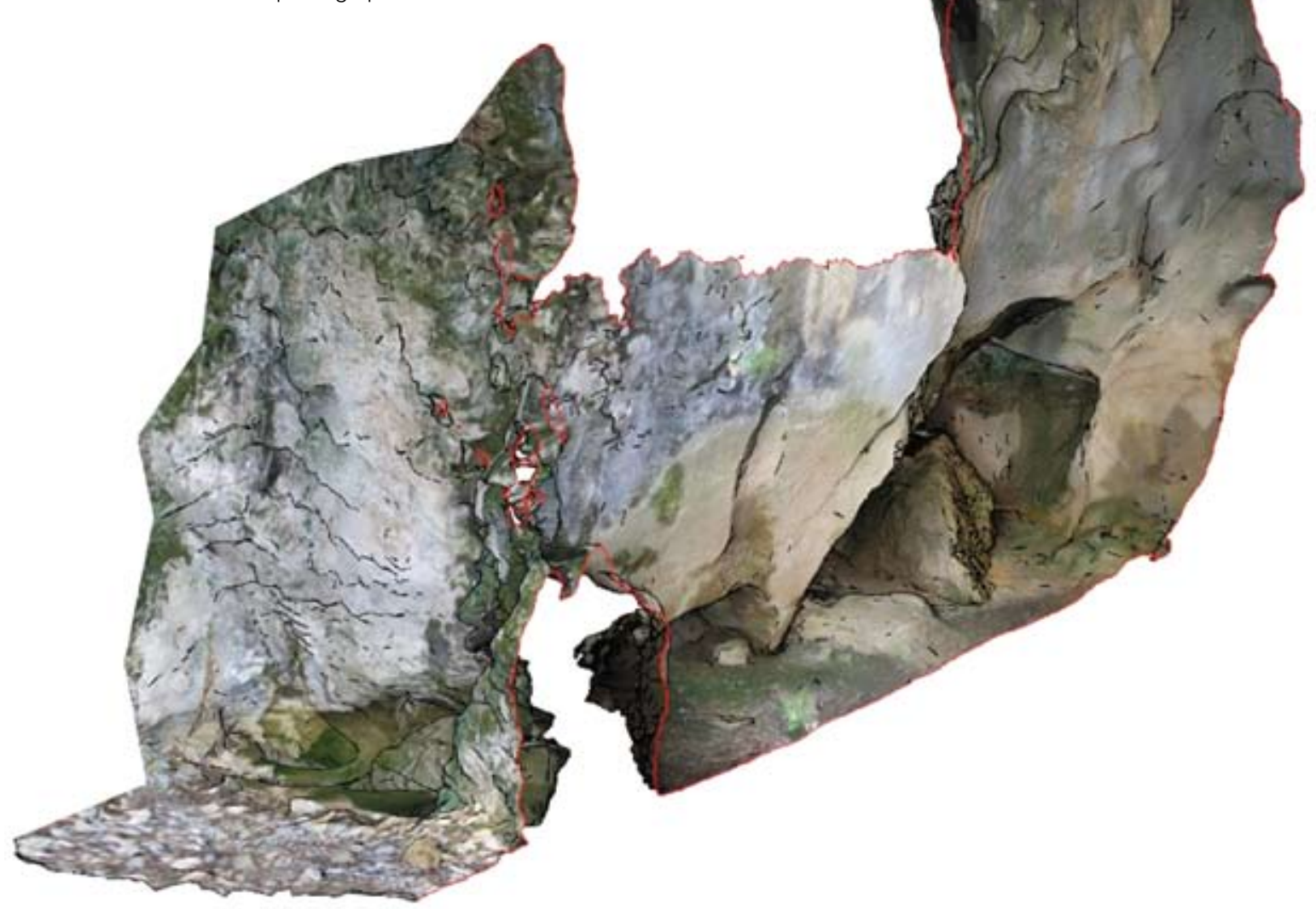

\section{5.- CONCLUSIONES}

Existen varias metodologías que pueden combinarse para las documentación geométrica de cavidades: topografía, fotogrametría, escáneres láser, etc., a partir de las cuales es posible obtener representaciones con un elevado nivel de detalle y realismo. Los productos permiten no sólo la representación cartográfica tradicional (plantas, secciones...) sino también acciones interactivas de difusión del patrimonio que faciliten el conocimiento de los lugares e incluso proporcionen la sensación de recorrerlos, incrementando así su aceptación como parte del patrimonio.

Tal como se ha desarrollado, a partir de las fotografías adquiridas en 2003 ha sido posible generar un nuevo conjunto de productos, entre los que destaca el modelo 3D con texturas fotográficas. Éste se ha generado a partir de las fotografías que se capturaron hace 10 años lo que, una vez más, nos recuerda la importancia de la correcta preservación de la información. Asimismo es destacable el hecho de que el avance de las tecnologías pone a nuestra disposición nuevas herramientas de procesamiento y análisis que permiten la obtención de nuevos resultados, si bien para que esto sea posible es preciso contar con datos de partida abundantes y de calidad.

\section{6.- BIBLIOGRAFÍA}

ALCALÁ ORTIZ, A., BERMÚDEZ CANO, R., RUIZ-RUANO COBO, F., BERMÚDEZ JIMÉNEZ, F.

2013 Espeleología y Arqueología. Cueva del Higueral-Guardia, ejemplo de un trabajo interdisciplinar. 1963-2013. Gota a gota 3, 42-51.

BOTELLA ORTEGA, D., BARROSO RUIZ, C., RIQUELME CANTAL, J.A., ABDESSADOK, S., CAPARRÓS, M., VERDÚ VERMEJO, L., MONGE GÓMEZ, G., GARCÍA SOLANO, J.A.

2006 La Cueva del Ángel (Lucena, Córdoba), un yacimiento del pleistoceno medio y superior del sur de la Península lbérica. Trabajos de Prehistoria 63-2, 153-165. 
BARRERA MAYO, S., BAEZA SANTAMARÍA, U.

2010 La Realidad Virtual aplicada a la explotación sostenible del Patrimonio Arqueológico. Un caso de éxito: la Cueva de Santimamiñe. Virtual Archaeology Review 1-1, 69-72.

BUCHÓN MORAGUES, F.F., HERRÁEZ BOQUERA, J.A., LERMA GARCÍA, J.L., PONS CRESPO, R., GALÍNDEZ HERÁNDEZ, M.

2002 Estudio fotogramétrico del abrigo de Cova Ribasals o Civil para la generación de la cartografía temática local y su análisis mediante sistemas de información geográfica. XIV Congreso Internacional de Ingeniería Gráfica (Santander, España), 11 pp. Disponible en: http://umw.cuevascastellon.uji.es/articulos/est_fotogrametrico_civil.pdf. (Acceso febrero de 2016).

CABRELLES LÓPEZ, M., LERMA GARCÍA, J.L.

2013 Documentación 3D de abrigos rupestres a partir de láser escáner y de procesos fotogramétricos automatizados. Virtual Archaeology Review 4-8, 64-68.

CORCHÓN RODRÍGUEZ, M.S., GÁRATE MAIDAGAN, D., GONZÁLEZ-AGUILERA, D., MUÑOZ NIETO, Á.L., GÓMEZ-LAHOZ, J., SABAS HERRERO, J.

2011 Nouveaux regards sur la Grotte de La Peña (San Román de Candamo, Asturies, Espagne). L'anthropologie 115, 384-424.

CÓRDOBA JIMÉNEZ, J.R., CAMACHO SÁNCHEZ, J., MESA MINGORANCE, J.L., RUIZ ARMENTEROS, A.

2004 Levantamiento topométrico y elaboración de la cartografía de la Cueva de los Murciélagos (Zuheros, Córdoba). VIII Congreso Nacional de Topografía y Cartografía TOPCART. Madrid. 2004. Disponible en: http://www. cartesia. org/geodoc/topcart2004/conferencias/18.pdf. (Acceso febrero de 2016).

FORTEA PÉREZ, J.

1990 Cuevas de la Lluera. Informe sobre los trabajos referentes a sus artes parietales. Excavaciones Arqueológicas en Asturias 1983-1986, 19-28.

FRYER, J.G., CHANDLER, J.H., EL-HAKIM, S.F.

2005 Recording and modelling an aboriginal cave painting: with or without laser scanning. ISPRS Archives- Volume XXXVI-5N17. Available at: http://www.isprs.org/proceedings/XXXVI/5-W17/pdf/3.pdf. (Acceso febrero de 2016).

GARCÍA LOZANO, F.J., LLANOS VIÑA, A.

1981 Levantamiento fotogramétrico del techo de la sala de las pinturas de la cueva de Altamira. Altamira Symposium, 590-612.

GÓMEZ-BARRERA, J.A., ORTEGA MARTÍNEZ, A.I., MARTÍN MERINO, A., FERNÁNDEZ MORENO, J.J., DEL VAL RECIO, J., GARCÍA DÍEZ, M., RUIZ GARCÍA, F., LATORRE GONZÁLEZ-MORO, P., CÁMARA MUÑOZ, L.

2001 Arte rupestre en el karst de Ojo Guareña (Merindad de Sotoscueva-Burgos): trabajos de documentación y estudio en «la Sala de la Fuente». Espacio, Tiempo y Forma, Serie I, Prehistoria y Arqueología 14, 203-226.
GONZÁLEZ-AGUILERA, D., MUÑOZ NIETO, Á.L., RODRÍGUEZGONZALVEZ, P., MENÉDEZ, M.

2011 New tools for rock art modelling: automated sensor integration in Pindal Cave. Journal of Archaeological Science $38,120-128$.

GRUSSENMEYER, P., LANDES, T., ALBY, E., CAROZZA, L.

2010 High resolution 3D recording and modelling of the Bronze Age cave "Les Fraux" in Perigord (France). ISPRS Archives - Volume XXXVIII, Part 5. Available at: http://unw.isprs. org/proceedings/XXXVIII/part5/papers/221.pdf. (Acceso febrero de 2016).

LDGP

2004 Documentación geométrica de la cueva de Praile Aitz I (Deba, Gipuzkoa). Laboratorio de Documentación Geométrica del Patrimonio (UPV/EHU). Disponible en: http:// hdl.handle.net/10810/14147 (acceso enero 2014).

LERMA, J.L., NAVARRO, S., CABRELLES, M., VILLAVERDE, V.

2010 Terrestrial laser scanning and close range photogrammetry for 3D archaeological documentation: the Upper Palaeolithic Cave of Parpalló as a case study. Journal of Archaeological Science 37, 499-507.

LOPETEGI GALARRAGA, A., VALLE MELÓN, J.M., RODRÍGUEZ MIRANDA, Á.

2004 Modelo de representación y difusión de cavidades. Cueva de Praileaitz. Deba, Gipuzkoa. VIII Congreso Nacional de Topografía y Cartografía TOPCART 2004 (Madrid, España). Disponible en: http://hdl.handle.net/10810/13765. (Acceso febrero de 2016).

NÚÑEZ, M.A., BUILL, F., EDO, M.

2013 3D model of the Can Sadurní cave. Journal of Archaeological Science 40, 4420-4428.

RIOS GARAIZAR, J., GARATE MAIDAGAN, D., REGALADO BUE$\mathrm{NO}, \mathrm{E}$.

2012 Excavación arqueológica en la cueva de Askondo (Mañaria, Askondo). Kobie Serie Bizkaiko Arkeologi Indusketak - Excavaciones Arqueológicas en Bizkaia 2, 31-38.

RUIZ REDONDO, A.

2010 Una revisión del Panel de las Manos de la cueva de El Castillo (Puente Viesgo, Cantabria). Munibe AntropologiaArkeologia 61, 17-27.

WARZÉE, N., GROENEN, M., ROSOUX, J., DEBEIR, O., ERCEK, R., REICHLING, C.

2008 Numérisation 3D de la grotte d'El Castillo (Puente Viesgo). En VERGNIEUX R. \& DELEVOIE, C. Actes du Colloque Virtual Restrospect 2007 (Bordeaux, France). Archéovision 3, 221-229. 
